\title{
The incidence of mesh extrusion after vaginal incontinence and pelvic floor prolapse surgery
}

\author{
Seth Cohen, Elizabeth Kavaler \\ Department of Urology, Lenox Hill Hospital, USA \\ Correspondence: Seth Cohen. Address: 100 E 77th St., N NY 10075, USA. E-mail: cohensethmd@gmail.com \\ Received: November 12, 2012 \\ Accepted: October 29, 2013 \\ Online Published: March 17, 2014 \\ DOI : $10.5430 /$ jha.v3n4p76 \\ URL: http://dx.doi.org/10.5430/jha.v3n4p76
}

\section{Abstract}

Purpose: The advantages of using synthetic mesh in vaginal reconstructive surgery are significant. However, the concern about extrusion has led many to question its use. We wished to learn the extrusion rates and time to extrusion in patients undergoing vaginal stress incontinence and prolapse surgeries using polypropylene mesh.

Materials and methods: Five hundred and seventy six women underwent vaginal reconstructive surgery with synthetic mesh between August 2000 and October 2009 for the treatment of stress urinary incontinence, with or without pelvic organ prolapse repair. 367 patients had at least one year follow-up. Procedures were: pubo-vaginal sling (PVS), PVS and anterior repair, PVS with anterior and/or posterior repairs, and PVS with hysterectomy and anterior and posterior repairs. Time to mesh extrusion was estimated using a survival function curve.

Results: Forty-two (11.4\%) patients sustained a mesh extrusion. The rate of mesh extrusion was (6.3\%) in the PVS group, (14.7\%) in the PVS and anterior repair group, (11.1\%) in the PVS with anterior and posterior repairs group and (5\%) in the PVS with hysterectomy and anterior and posterior repair. Percent extrusion free at one to four years post-op was (91\%) and (85\%).

Conclusion: Our study provides a large series of prolapse cases performed by a single surgeon with follow-up that extends at least one year, with the longest follow-up at eight years. The incidence of long term mesh extrusion needs to be considered with respect to the support advantages of synthetic mesh in planning vaginal reconstructive surgery.

\section{Key words}

Pubo-vagina sling, Vaginal mesh, Pelvic floor mesh, Mesh extrusion, Mesh erosion

\section{Introduction}

The concept of a suburethral sling for the management of stress urinary incontinence (SUI) was first introduced in the early 1900s utilizing the gracilis muscle ${ }^{[1]}$. Since then, various other autologous harvest sites have been identified, resulting in durable outcomes ${ }^{[2]}$. However, harvest site morbidities, including pain, difficulty with ambulation, cosmetic issues, and long recovery times, have led to the introduction of other materials ${ }^{[3]}$. Currently, synthetic materials have gained widespread acceptance for use in prolapse repair because of lack of harvest site morbidities, relative ease in placement, and the durability of the tension-free support that they provide ${ }^{[4,5]}$. Recently, the use of synthetic mesh for vaginal reconstructive surgery has created concern, prompted by an FDA warning regarding the risk of extrusion. 
Synthetic mesh has at least three broad purposes for use in pelvic floor reconstruction: (1) to substitute where tissue is lacking, (2) to reinforce a weak pelvic floor and (3) to generate new supportive tissue ${ }^{[6]}$. Proponents of synthetics have argued that the tissues in women with prolapse are inherently weak and imbrication of those tissues alone cannot provide adequate support for repair. With an increase in the utilization of synthetic mesh for pelvic floor surgery, concerns have been raised regarding graft extrusion into the vagina and/or urinary tract often requiring additional surgery ${ }^{[7]}$. We present our extensive experience with the use of synthetic mesh in vaginal reconstruction and report our rates of mesh extrusion with follow-up of at least one year from surgery.

\section{Materials and methods}

Five hundred and seventy six consecutive women underwent vaginal reconstructive surgery with synthetic mesh between August 2000 and October 2009 by a single surgeon (EAK) at a single institution (Lenox Hill Hospital, Department of Urology in Manhattan, NY).

Every patient had non-absorbable polypropylene slings placed, either with or without concomitant pelvic organ prolapse repair, all of which were performed using synthetic mesh (Prolift, Johnson and Johnson) or (Anterior/Posterior Elevate, AMS). Three hundred and sixty seven patients in the study period had at least one year follow-up and constitute the study subjects.

The pelvic surgeries were divided into four groups: 63 patients underwent a polypropylene pubo-vaginal sling (PVS) alone, 149 underwent a PVS with anterior repair, 135 underwent PVS with anterior and posterior repairs, and 20 underwent PVS with vaginal hysterectomy and anterior and posterior repairs. The amount of mesh used was dependent upon the type of defect: anterior, posterior, and/or apical.

Before surgery, all patients underwent a complete history and physical examination, Marshall Test, multichannel videourodynamic exam, and filled out an Incontinence Quality of Life Questionnaire (see Figure 1). All patients received intraoperative antibiotics, typically a first generation cephalosporin like Cefazolin.

Figure 1. Incontinence quality of life questionnaire

Note. ALB-avoidance and limiting behavior; PSI-psychosocial impacts; SD-standard deviation; SE-social embarrassment

\begin{tabular}{|l|}
\hline ALB domain \\
I worry about not being able to get to the toilet on time \\
I worry about coughing/sneezing because of my incontinence \\
I have to be careful standing up from sitting \\
I worry about where toilets are in new places \\
It's important for me to make frequent trips to the toilet \\
It's important to plan every detail in advance because of my incontinence \\
I have difficulty getting a good night's sleep because of my incontinence \\
I have to watch how much I drink because of my incontinence \\
PSI domain \\
I feel depressed because of my incontinence \\
I don't feel free to leave home for long periods because of my incontinence \\
I feel frustrated because my incontinence prevents me doing what I want \\
My incontinence is always on my mind \\
My incontinence makes me feel unhealthy \\
My incontinence makes me feel helpless \\
I get less enjoyment out of life because of my incontinence \\
My incontinence limits my choice of clothing \\
I worry about having sex because of my incontinence \\
SE domain \\
I worry about others smelling urine on me \\
I worry about my incontinence getting worse as I get older \\
I worry about being embarrassed or humiliated by my incontinence \\
I worry about wetting myself \\
I feel I have no control over my bladder
\end{tabular}


For postoperative care, estrogen cream was prescribed for vaginal application for one month. Post-operative oral antibiotics were not routinely prescribed. Any patient who experienced irritative voiding symptoms, suprapubic pain, urinary tract infections, vaginal discharge and spotting, or noticed a foreign body upon manual palpation were instructed to come in to our office for immediate evaluation. Extrusions were confirmed by physical examination alone. In these cases local excision of the mesh was performed in an ambulatory setting under anesthesia. The vaginal mucosa was undermined, the exposed mesh removed and the mucosa was either left open to heal by secondary intention or closed loosely with interrupted vicryl sutures.

A Kaplan-Meier survival curve was generated to compare differences in the time to mesh extrusion among the various procedures. Our goal was to determine if mesh volume or length of time from surgery played a role in the occurrence of mesh extrusion. Statistical analysis was performed with SAS (SAS Institute, Cary, NC) statistical software.

\section{Results}

None of the 367 patients experienced urinary tract erosions; all mesh extrusions were through the vaginal wall only. Overall, mesh extrusion was observed in 42 of the 367 patients (11.4\%). The rate of mesh extrusion was 6.3\% (4/63) in the PVS group, 14.7\% (22/149) in the PVS and anterior repair group, 11.1\% (15/135) in the PVS with anterior and posterior repairs group and 5\% (1/20) in the PVS with hysterectomy and anterior and posterior. A difference in time to mesh extrusion was also observed among the various procedures. The median number of months to mesh extrusion was 2.6 months (range three months to six months) in the PVS group, four months (range 20 days to seven years) in the PVS and anterior repair group, two months (range 14 days to 17 months) in the PVS with anterior and posterior repairs group and 24 months in the PVS with hysterectomy and anterior and posterior repairs group. Age and incidence of co-morbidities like diabetes and hypertension were equally distributed throughout the entire cohort.

Figure 2 is survival function curve demonstrating time to mesh extrusion. Percent extrusion free at one year post-operative was $91 \%$, at two years $89 \%$, at three years $87 \%$ and at four years $85 \%$. An increasing number of patients were lost to follow up the farther out from their surgery and thus the sample size dramatically decreased past five years follow up making it difficult to draw any valid conclusions beyond this point.

Figure 2. Kaplan-Meier survival function curve showing time to mesh extrusion with all procedures

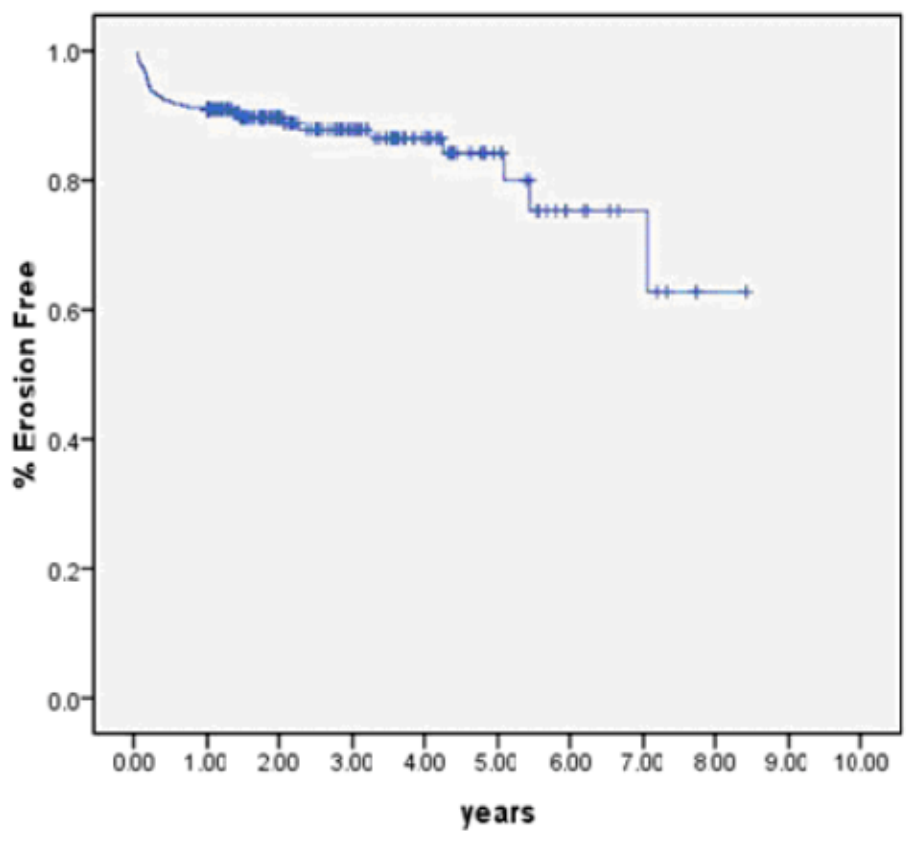

ISSN 1927-6990 E-ISSN 1927-7008 
No statistically significant difference was seen between the four groups with regard to the incidence of, or time to, mesh extrusion. In addition, there were no patterns to the location of mesh extrusions. The amount of mesh used did not impact on the risk of extrusion. However, the greatest numbers of extrusions: 23 (55\%) were seen before 90 days follow up; followed by 10 extrusions (24\%) up to one year follow up, six (14\%) up to five years follow up and three (7\%) at greater than five years follow up (see the Table).

Table. Results based on time to extrusion

\begin{tabular}{lll}
\hline Follow up & Extrusion (42) & Incidence of Extrusion (\%) \\
\hline 90 days & 23 & 55 \\
91 days - one year & 10 & 24 \\
one year - five years & 6 & 14 \\
$>$ five years & 3 & 7 \\
\hline
\end{tabular}

\section{Discussion}

The pathophysiology of stress incontinence has been described as a global laxity of pelvic fascial support secondary to an inherent deficiency in elastin, collagen, and skeletal muscle fibers ${ }^{[8]}$. Similarly, other authors have observed tears or breaks in the endopelvic fascia of women with pelvic floor prolapse during reconstructive surgery ${ }^{[9]}$. Regardless of the etiology of the laxity, many reconstructive surgeons have argued that prolapsed vaginal tissue is weak and, alone, cannot provide support for adequate repair, creating a need for further tissue reinforcement ${ }^{[10]}$. The current available options for graft materials applicable to pelvic floor support include autologous fascia, cadaveric fascia, porcine dermis, porcine small intestine submucosa, and synthetic mesh ${ }^{[11]}$. Each of these materials has its advantages and disadvantages. In order for patients and surgeons to make informed choices, they must have accurate and reliable data regarding the risks and benefits of each option. We provide our results with synthetic mesh in order to address the risk of extrusion in a cohort of patients who underwent surgeries for various degrees of prolapse repair at a single institution by a single surgeon. It is one of the largest series looking at mesh extrusion in vaginal pelvic reconstructive surgery with follow-up of at least one year.

The concept of the pubovaginal sling for the treatment of stress urinary incontinence was introduced in the early 1900s with the use of autologous gracilis muscle ${ }^{[12]}$. Sixty five years later the first synthetic slings were created ${ }^{[13]}$. Currently, the most commonly used synthetic material is loosely woven, polypropylene mesh. Major advantages include better dry rates, and avoidance of tissue harvesting ${ }^{[3]}$. In 1999, two authors first reported that the biomechanical properties of synthetic material are superior to those of autologous tissues ${ }^{[14]}$. The recent updated AUA Guidelines on the surgical management of female stress urinary incontinence reported estimated cure/dry rates for patients undergoing cadaveric sling procedures of 74\%-80\% during a 12 months - 48 months follow-up period. The estimated cure/dry rates for midurethral synthetic slings were $81 \%-84 \%$ at 12 months -48 months ${ }^{[15]}$. With similar dry rates, the question remains whether the improved pelvic floor support with the synthetic slings translates into better long-term durability without increasing morbidity ${ }^{[13]}$. The use of synthetic mesh in grafting for prolapse surgery is less widely accepted because of concerns regarding extrusion.

Over the past three years, the FDA has received over 1,000 reports from surgical mesh manufacturers of complications that were associated with the use of polypropylene mesh in vaginal pelvic organ prolapse repair and pubovaginal slings for the treatment of stress incontinence ${ }^{[16]}$. The most frequent such complications included extrusion through the vaginal epithelium, infection, pain, contracture, urinary problems, and recurrence of prolapse and/or incontinence. There were also reports of bowel, bladder, and blood vessel perforation during insertion. Contributing factors to extrusion may include the overall health of the patient, the mesh material, the size and shape of the mesh, the surgical technique used, concomitant procedures undertaken (e.g. hysterectomy), and possibly estrogen status ${ }^{[16]}$. 
Long term data to adequately assess the risk of extrusion in both prolapse repair and sling surgery is not available, and short term rates of vaginal extrusion in the literature are inconsistent. Most trials focus on one reconstructive technique, or review results with a new mesh often providing very short follow-up. Consequently, it is difficult to draw definitive conclusions about the safety and efficacy of mesh utilized for vaginal reconstruction.

The rate of mesh extrusion into the vagina and or erosion into urinary tract is the complication of greatest concern limiting its use among many pelvic reconstructive surgeons. Our success rates using synthetic mesh to treat stress incontinence either with or without concomitant pelvic floor repair have been similar to those quoted by others. Mesh extrusion has been reported at a rate of $2.8 \%$ to $11 \%$ using synthetic mesh for the correction of anterior vaginal wall prolapse ${ }^{[17]}$. Petros et al. looked at 85 patients who underwent vaginal reconstructive surgery utilizing synthetic mesh and found an $81 \%$ extrusion free at four years ${ }^{[18]}$. Using a long-term time to mesh extrusion analysis, we were able to assess vaginal extrusion rates in our study population. $11.4 \%$ of patients overall experienced extrusion at any given time, whereas $91 \%$ and $85 \%$ of patients that were available for follow up at one to four years were extrusion free.

There were no trends seen when comparing the four different surgical groups. One would expect to see greater extrusion rates in procedures that involve more aggressive dissection as well as those that incorporate a greater amount of mesh to repair these larger defects. But, this was not the case. A prospective, randomized study distributed evenly among the four groups would help to sort out this concern. Some authors have proposed observing vaginal mesh extrusions that do not involve the urinary tract ${ }^{[19]}$. Kobashi and Govier hypothesized that the porous nature of polypropelene allows for re-epithelialization of eroded mesh and in-growth into surrounding tissue ${ }^{[19]}$. In their study, population extruded mesh of up to $1 \mathrm{~cm}$ re-epitheliazed within six weeks. Typically, patients with extruded mesh are too uncomfortable and discouraged to be placed on conservative management. In our experience with vaginal mesh extrusion, re-epitheliazation usually does not completely cover the entirety of the mesh necessitating surgical removal. Vaginal extrusions are usually due to subclinical infections or disruption of the suture line by hematoma or trauma ${ }^{[3]}$. Thus, for those reasons, our practice is to perform immediate surgical excision in patients presenting with vaginal mesh extrusion.

One limitation of this study is that no consistent grading scale for location and extent of mesh extrusion exists. The development of an extrusion grading scale would allow for an easier comparison of extrusion rates among different studies. Lastly, longer patient follow-up might yield statistical significance in the comparison of time to mesh extrusion in the four different procedure arms.

\section{Conclusion}

Our study provides a large series of prolapse cases performed by a single surgeon with follow-up that extends at least one year, with the longest follow-up at eight years. The results show our extrusion rate at one to eight years after mesh repair for stress urinary incontinence in combination with anterior, posterior and/or hysterectomy is $11.4 \%$. We determined that pelvic floor reconstructive surgeons can expect higher extrusion rates early in the post-operative recovery period, and decreased extrusion rates the further out from surgery. Our study offers valuable information to surgeons contemplating the use of mesh in vaginal reconstructive surgery.

We consider $11.4 \%$ an acceptable rate of extrusion when compared to the current literature. There are a myriad of mesh kits out on the market which vary from non-absorbable polypropylene mono-filament mesh to biologic-polypropylene mesh with absorbable properties. Since the risk of recurrence is higher when mesh isn't used to repair pelvic organ prolapse, we would rather take the risk of extrusion over the risk of recurrence. In accordance with the new FDA recommendations, the incidence of long-term mesh extrusion needs to be considered with respect to the support advantages of synthetic mesh in planning vaginal reconstructive surgery. Informed consent regarding the risk of extrusion is an important factor in counseling patients regarding their choice of reinforcing materials. As more pelvic surgeons adopt the use of mesh in their surgeries, techniques and mesh materials will improve. With more experience, we should be able to reduce the risk of extrusion even further. 


\section{References}

[1] Flisser A, Blaivas J. Outcome of urethral reconstructive surgery in a series of 74 women. J Urol. 2003; 169: 2246-2249. PMid: 12771761. http://dx.doi.org/10.1097/01.ju.0000061763.88247.16

[2] Carbone JM, Kavaler E, Hu JC. Pubovaginal sling using cadaveric fascia and bone anchors: disappointing early results. J Urol. 2001; 165(5): 1605-1611. http://dx.doi.org/10.1016/S0022-5347(05)66358-6

[3] Siegel A., Kim M, Goldstein M. High incidence of vaginal mesh extrusion using the intravaginal slingplasty, J Urol. 2005; 174: 1308-1311. PMid: 16145409. http://dx.doi.org/10.1097/01.ju.0000173927.74235.e9

[4] Hill JR, Isom-Batz, Kavaler E. Patient perceived outcomes of treatments used for interstitial cystitis. Urology. 2008; 71(1): 62-66. PMid: 18242366. http://dx.doi.org/10.1016/j.urology.2007.09.011

[5] Petros, PP. The intravaginal slingplasty operation, a minimally invasive technique for cure of urinary incontinence in the female. Aust N Z J Obstet Gynaecol. 1996; 36: 453-461. http://dx.doi.org/10.1111/j.1479-828X.1996.tb02192.x

[6] Baessler K. Maher, C. Mesh augmentation during pelvic-floor reconstructive surgery: risks and benefits. Curr Opin Obstet Gynecol. 2006; 18: 560-566. PMid: 16932053. http://dx.doi.org/10.1097/01.gco.0000242961.48114.b0

[7] Kobashi K. Management of erosion of graft materials in pelvic floor reconstruction. ScientificWorldJournal. 2009; 9: 32-36. PMid: 19151896. http://dx.doi.org/10.1100/tsw.2009.2

[8] Jung HJ, Yim GW, Jeon MJ. Preoperative maximum urethral closure pressure and valsalva leak point pressure as predictive parameters for midurethral sling. J Reprod Med. 2009; 54(7): 436-40. PMid: 19691260.

[9] Richardson AC, Lyon JB, Williams NL. A new look at pelvic relaxation. Am J Obstet Gynecol. 1976; 126: 568-573. PMid: 984127.

[10] Cervigni M, Natale F. The use of synthetics in the treatment of pelvic organ prolapse. Curr Opin Urol. 2001; 11: 429-435. http://dx.doi.org/10.1097/00042307-200107000-00016

[11] Dora CD, Dimarco DS, Zobitz ME. Time dependent variations in biomechanical properties of cadaveric fascia, porcine dermis, porcine small intestine submucosa, polypropylene mesh and autologous fascia in the rabbit model: Implications for sling surgery. $\mathrm{J}$ Urol. 2004; 171: 1970-1973. PMid: 15076323. http://dx.doi.org/10.1097/01.ju.0000121377.61788.ad

[12] Westney OL, McGuire EJ. Pubovaginal Slings. Hurt WG editors. Urogynecologic Surgery, 2nd edition. Philadelphia. Lippincott Williams \& Wilkins CO. 2000; chap.5-5: 93.

[13] Choe JM, Ogan K, Battino BS. Antimicrobial mesh versus vaginal wall sling: A compariative outcomes analysis. J Urol. 2000; 163: 1829-1834. http://dx.doi.org/10.1016/S0022-5347(05)67553-2

[14] Corujo M, Badlani GH. Epithelialization of permanent stents. J Endourol. 1997; 11: 477-480. http://dx.doi.org/10.1089/end.1997.11.477

[15] Dmochowski RR, Blaivas JM, Gormley EA. Update of AUA guidline on the surgical management of female stress urinary incontinence. J Urol. 2010; 183(5): 1906-1914. PMid: 20303102. http://dx.doi.org/10.1016/j.juro.2010.02.2369

[16] FDA Public Health Notification: Serious Complications Associated with Transvaginal Placement of Surgical Mesh in Repair of Pelvic Organ Prolapse and Stress Urinary Incontinence. Issued: October 20, 2008.

[17] Jo H., Kim JW, Park NH. Efficacy and outcome of anterior vaginal wall repair using polypropylene mesh (Gynemesh). J Obstet Gynaecol. 2007; 33: 700-704.

[18] Petros, PP. Medium-term follow-up of the intravaginal slingplasty operation indicates minimal deterioration of urinary continence with time. Aust N Z J Obstet Gynaecol. 1999; 39: 354-356. http://dx.doi.org/10.1111/j.1479-828X.1999.tb03414.x

[19] Kobashi KC, Govier FE. Management of vaginal erosion of polypropylene mesh slings. J Urol. 2003; 169: $2242-2243$. 